\title{
Imaging the pelvic floor
}

\author{
G. A. Santoro ${ }^{1}$
}

Received: 17 July 2017/ Accepted: 18 July 2017/Published online: 3 August 2017

(C) Springer International Publishing AG 2017

Urinary and fecal incontinence, obstructed defecation, pelvic pain and pelvic organ prolapse are common in older multiparous women, affecting approximately $16 \%$ of females aged 40-56 years [1]. As we evaluate a patient with a clinically evident anatomic defect (rectocele, rectal prolapse, perineal descent), it is essential to establish whether this defect is really associated with the patient's symptoms (obstructed defecation). Moreover, a pelvic floor disorder in one compartment frequently coexists with disorders involving other compartments. Indeed, rather than considering the pelvic floor as divided into three vertical compartments, the current concept is to approach the pelvic floor as a horizontal structure [1]. The pelvic floor is a three-dimensional mechanical apparatus that acts as a unit, with a close interrelationship between organ systems, fascia and ligaments, muscles, vessels and nerves. Because the levator ani muscle provides support to all three organ systems, its weakness will result in impaired function of any, or all, of the structures that the muscle supports. In addition, damage to the endopelvic, pubocervical or rectovaginal fascias will lead to herniation of one organ system into another. This is demonstrated by the common coexistence of prolapse of the anterior and posterior vaginal walls requiring repair of both. Understanding pelvic floor anatomy is therefore crucial for an effective management of these dysfunctions.

A careful preoperative evaluation is key to determining the pathophysiology of pelvic floor dysfunction [2]. Clinical examination, however, is not accurate in diagnosing

\footnotetext{
G. A. Santoro

giulioasantoro@yahoo.com

1 Pelvic Floor Unit, I'Department of Surgery, Regional Hospital, Treviso, Italy
}

anatomical defects of the posterior vaginal wall, overestimating the presence of rectocele (large false-positive rate) but missing enterocele or intussusception in patients with primary pelvic organ prolapse (large false-negative rate) $[3,4]$. Imaging allows the clinician to better evaluate the patients in order to determine what anatomical alterations are present, and this leads to appropriate surgical interventions and increased success rates [5]. Traditional evacuation proctography or colpocystoproctography, modern dynamic magnetic resonance imaging and ultrasound can be used for visualization of the pelvic floor [5]. Advantages of ultrasound over the other imaging modalities are that it is more widely available, more cost-effective, less time-consuming, and associated with better patient compliance. It is performed by the clinician during office consultation and can be considered as an extension of the physical examination. The development of new technological innovations such as three-/four-dimensional (3D/4D) tools, video-recording, post-processing analysis and the standardization of methodology and terminology [6] have made ultrasound imaging more reproducible and less operator dependent.

Several ultrasound techniques can be used to assess the pelvic floor [6]. Translabial ultrasound (TLUS) is performed with the patient placed in the dorsal lithotomy position, with hips flexed and abducted, by using a convex conventional transducer (with frequencies of 3-6 MHz and field of view at least $70^{\circ}$ ) positioned on the perineum. TLUS provides two-dimensional (2D) imaging of the pelvic floor. In the midsagittal plane, all anatomical structures (bladder, urethra, vaginal walls, anal canal and rectum) between the posterior surface of the symphysis pubis and the posterior part of the levator ani are visualized [7]. Imaging is usually performed with the patient at rest, during maximal Valsalva maneuver and during pelvic floor 
muscle contraction (PFMC). This dynamic modality allows assessment of pelvic organ prolapse, perineal descent or pelvic floor dyssynergy. Using 3D volumetric probes developed for obstetric imaging, 3D- and 4D-TLUS may be performed [7]. An advantage of this technique, compared with $2 \mathrm{D}$ mode, is the opportunity to obtain tomographic or multislice imaging, for example in the axial plane, in order to assess the entire puborectalis muscle and its attachment to the pubic rami. It is also possible to measure the diameter and area of the levator hiatus and to determine the degree of hiatal distension on Valsalva maneuver [8]. 4D imaging involves real-time acquisition of volume ultrasound data, which can then be visualized instantly in orthogonal planes or rendered volumes.

Endoanal ultrasound (EAUS) is performed with a high multifrequency, $360^{\circ}$ rotational mechanical probe which allows automatic 3D acquisition [9]. During examination, the patient may be placed in a dorsal lithotomy, left lateral or prone position. EAUS is firmly established as the "gold standard" modality for investigating internal and external sphincter integrity and detecting undiagnosed obstetric anal sphincter injuries (OASIS) $[10,11]$. It can be useful in the management of pregnancies following OASIS [12] and to evaluate the results of treatment (anterior sphincter repair, bulking agent injections).

Endovaginal ultrasound (EVUS) is performed with the patient placed in the dorsal lithotomy position. By using the same $360^{\circ}$ rotational mechanical probe as used for EAUS, this procedure provides 3D high-resolution images of the anatomical structures of the pelvic floor [13]. It allows assessment of female urethral complex [14], biometric indices of the levator hiatus [15], damage of the levator ani [16], endopelvic fascia and/or the perineal body [17].

Echodefecography (EDF) is a relatively new technique for evaluating posterior compartment prolapse [18]. It is a 3D dynamic anorectal ultrasound modality performed with the same $360^{\circ}$ rotating transducer used for EAUS. Patients are examined in the left lateral position, with the probe inserted into the rectum. Images are acquired by four automatic scans: scan 1: at rest position without gel; scan 2: at rest-straining-at rest without gel; scan 3: the transducer is positioned proximally to the anorectal junction in fixed position and does not follow the descending muscles of the pelvic floor during maximum straining; scan 4: at reststraining-at rest following introduction of $60-120 \mathrm{ml}$ ultrasound gel into the rectal ampulla. The purpose of the scan is to visualize and quantify all anatomical structures and functional changes associated with straining (rectocele, intussusception, grade II or III sigmoidocele/enterocele). However, compared to TLUS or EVUS, the presence of the transducer inside the rectum can limit or block the full descent of the rectal wall during a Valsalva maneuver and this is a source of a possible false negative in patients with intussusception or internal prolapse.

Given the wealth of accumulating evidence, as previously reported, the question is not whether we should scan the pelvic floor, but rather what is the best way to scan the pelvic floor. Division into anterior, middle and posterior compartments has led to fragmentation of assessment: The anterior and middle compartments have been the domain of urogynecologists, who commonly use 2D/3D TLUS and EVUS as their modality of choice for scanning, whereas the posterior compartment is traditionally assessed by colorectal surgeons, who prefer 3D-EAUS and EDF. These artificial divisions of the pelvis, however, fail to recognize the close anatomical and functional relationship of these three compartments. For this reason, we introduced the "integrated, multicompartmental" ultrasonographic approach [6]. According to the patient's symptoms and clinical evaluation, the clinicians may use a different combination of ultrasound techniques (TLUS, EVUS, EAUS and EDF) to complement the advantages and to overcome the limitations of each of these modalities and to substantially improve the clinical management of PFD. An example of this integrated approach is presented by Regadas et al. in their paper entitled "Dynamic translabial ultrasound versus echodefecography combined with the endovaginal approach to assess pelvic floor dysfunctions: how effective are these techniques?" featured in this issue of Techniques in Coloproctology [19]. The authors used 3D-EVUS to evaluate the anatomical integrity of the pubovisceral muscle and detect complete or partial detachment of the muscle from the pubic rami. Functional aspects were assessed with EDF, used to evaluate the movement of the puborectalis muscle during straining in order to identify a paradoxical contraction (anismus), to quantify the perineal descent and to measure the depth of rectocele, intussusception, enterocele or cystocele. They reported good correlation between EDF and TLUS in the identification of pelvic organ prolapse and anismus. Additionally, the main advantage of combining 3D-EVUS to EDF was the possibility to identify anal sphincters and pubovisceral muscle defects that TLUS was not able to demonstrate.

Integrated total pelvic floor ultrasound is becoming the standard of care, along with the multidisciplinary team approach to the evaluation and treatment of pelvic floor dysfunction [20-22].

\section{Compliance with ethical standards}

Conflict of interest The authors declare that they have no conflict of interest.

Ethical approval Ethical approval is not required for this paper. 
Informed consent For this type of study, formal consent is not required.

\section{References}

1. Davila GW, Ghoniem GM (2003) Pelvic floor dysfunction: the importance of a multidisciplinary approach. Clin Colon Rectal Surg 16:3-4. doi:10.1055/s-2003-39031

2. Tubaro A, Vodušek DB, Amarenco G, et al (2013) Imaging, neurophysiological testing and other tests. In: Abrams P, Cardozo L, Khoury S, Wein A (eds) ICUD-EAU 5th international consultation on incontinence, chapter 7. ICUD-EAU, Paris, pp 507-622

3. Marques da Silva G, Gurland B, Sleemi A, Levy G (2006) Posterior vaginal wall prolapse does not correlate with fecal symptoms or objective measures of anorectal function. Am J Obstet Gynecol 195:1742-1747. doi:10.1016/j.ajog.2006.07.034

4. Groenendijk AG, van der Hulst VP, Birnie E, Bonsel GJ (2008) Correlation between posterior vaginal wall defects assessed by clinical examination and by defecography. Int Urogynecol $\mathbf{J}$ 19:1291-1297. doi:10.1007/s00192-008-0632-9

5. Groenendijk AG, Birnie E, de Blok S et al (2009) Clinical-decision taking in primary pelvic organ prolapse; the effects of diagnostic tests on treatment selection in comparison with a consensus meeting. Int Urogynecol J Pelvic Floor Dysfunct 20:711-719. doi:10.1007/s00192-009-0846-5

6. Santoro GA, Wieczorek AP, Dietz HP et al (2011) State of the art: an integrated approach to pelvic floor ultrasonography. Ultrasound Obstet Gynecol 37:381-396. doi:10.1002/uog.8816

7. Dietz HP, Steensma AB (2005) Posterior compartment prolapse on two-dimensional and three-dimensional pelvic floor ultrasound: the distinction between true rectocele, perineal hypermobility and enterocele. Ultrasound Obstet Gynecol 26:73-77. doi:10.1002/uog.1930

8. Dietz HP, Shek C, Clarke B (2005) Biometry of the pubovisceral muscle and levator hiatus by three-dimensional pelvic floor ultrasound. Ultrasound Obstet Gynecol 25:580-585. doi:10.1002/ uog. 1899

9. Santoro GA, Fortling B (2007) The advantages of volume rendering in three-dimensional endosonography of the anorectum. Dis Colon Rectum 50:359-368. doi:10.1002/uog.1899

10. Bliss DZ, Mellgren A, Whitehead WE, et al (2013) Assessment and conservative management of faecal incontinence and quality of life in adults. In: Abrams P, Cardozo L, Khoury S, Wein A (eds) ICUD-EAU 5th international consultation on incontinence, chapter 16. ICUD-EAU, Paris, pp 1443-1486

11. Sioutis D, Thakar R, Sultan AH (2016) Over diagnosis and rising rates of Obstetric Anal Sphincter Injuries (OASIS) - time for reappraisal. Ultrasound Obstet Gynecol. doi:10.1002/uog.17306
12. Cerro CR, Franco EM, Santoro GA et al (2017) Residual defects after repair of obstetric anal sphincter injuries and pelvic floor muscle strength are related to anal incontinence symptoms. Int Urogynecol J 28:455-460. doi:10.1007/s00192-016-3136-Z

13. Santoro GA, Wieczorek AP, Stankiewicz A et al (2009) Highresolution three-dimensional endovaginal ultrasonography in the assessment of pelvic floor anatomy: a preliminary study. Int Urogynecol J Pelvic Floor Dysfunct 20:1213-1222. doi:10.1007/ s00192-009-0928-4

14. Wieczorek AP, Wozniak MM, Stankiewicz A et al (2012) 3-D high-frequency endovaginal ultrasound of female urethral complex and assessment of inter-observer reliability. Eur J Radiol 81:e7-e12. doi:10.1016/j.ejrad.2010.09.044

15. Santoro GA, Wieczorek AP, Shobeiri SA et al (2011) Interobserver and interdisciplinary reproducibility of 3D endovaginal ultrasound assessment of pelvic floor anatomy. Int Urogynecol J 22:53-59. doi:10.1007/s00192-010-1233-y

16. Rostaminia G, Manonai J, Leclaire E et al (2014) Interrater reliability of assessing levator ani deficiency with $360^{\circ} 3 \mathrm{D}$ endovaginal ultrasound. Int Urogynecol J 25:761-766. doi:10. 1007/s00192-013-2286-5

17. Santoro GA, Shobeiri SA, Petros PP, Zapater P, Wieczorek AP (2016) Perineal body anatomy seen by three-dimensional endovaginal ultrasound of asymptomatic nulliparae. Colorectal Dis 18:400-409. doi:10.1111/codi.13119

18. Murad-Regadas SM, Regadas FSP, Rodrigues LV et al (2008) A novel three-dimensional dynamic anorectal ultrasonography technique (echodefecography) to assess obstructed defecation, a comparison with defecography. Surg Endosc 22:974-979. doi:10. 1007/s00464-007-9532-1

19. Murad-Regadas SM, Karbage SA, Bezerra LS, Regadas FSP, da Silva Vilarinho A, Borges LB, Regadas Filho FSP, Veras LB (2017) Dynamic translabial ultrasound versus echodefecography combined with the endovaginal approach to assess pelvic floor dysfunctions: How effective are these techniques? Tech Coloproctol. doi:10.1007/s10151-017-1658-0

20. Hainsworth AJ, Solanki D, Schizas AM, Williams AB (2015) Total pelvic floor ultrasound for pelvic floor defaecatory dysfunction: a pictorial review. $\mathrm{Br} \mathrm{J}$ Radiol. doi:10.1259/bjr. 20150494

21. Hainsworth AJ, Pilkington SA, Grierson C et al (2016) Accuracy of integrated total pelvic floor ultrasound compared to defaecatory MRI in females with pelvic floor defaecatory dysfunction. $\mathrm{Br}$ J Radiol. doi:10.1259/bjr.20160522

22. Lone F, Sultan AH, Stankiewicz A, Thakar R (2014) The value of pre-operative multicompartment pelvic floor ultrasonography: a 1-year prospective study. Br J Radiol. doi:10.1259/bjr.20140145 